\title{
Numerical study of spherical flame in shock accelerated flows with different reflected shock wave distances
}

\author{
Yuejin Zhua , Lei Yu and Lu Dai \\ School of Energy and Power Engineering , Jiangsu University, Zhenjiang 212013 , Jiangsu , \\ China \\ azyjwind@163.com
}

\begin{abstract}
Keywords: shock, flame, Richtmyer-Meshkov instability, reflected shock wave distance
Abstract. Flame distortion induced by shock waves frequently occurs in natural and human activities. To deeply understand the characteristics of flame distortion and combustion, a two-dimensional numerical study of a spherical flame distortion induced by a planar incident shock wave and its reflected wave was carried out for an ethylene/oxygen/nitrogen mixture. And a single-step chemical reaction and high resolution of grid was adopted. It can be found that before the interaction of reflected shock wave with flame, the physical process plays an important role; while after the interaction between reflected shock wave and distorted flame, the reaction heat release rate and flame interface length increase quickly, and the controlling mechanism of flame distortion is transforming to the chemical reaction process. Moreover, the longer reflected shock wave distance induces that the influence of viscosity is stronger.
\end{abstract}

\section{Introduction}

The phenomenon of shock wave interacts with a flame frequently occurs in natural and human activities, such as supersonic combustion propulsion applications [1] and industrial explosions [2]. Besides, it also involves many complicated physics and chemical processes. Thus, the related research is practically significant.

As a basic problem in compressible reactive flow, study of shock-flame interaction has been widely investigated experimentally and numerically. Ju et al. [3] investigated the influence of different mach number on flame evolution. And Thomas et al. [4] experimentally studied the shock-flame interaction, and found that Richtmyer-Meshkov (RM) instability is the main mechanism of controlling the distortion and instability of flame. While Oran et al. [2] carried out a series of numerical studies on incident shock wave and flame interaction, and they found there would occur hot spots even detonation under proper flow conditions. In recent years, we also $[5,6]$ published some related works. However, there still exists much unclear information during the whole shock-flame interaction process owing to its complicated and transient characteristics. For instance, different shock reflected distance can induce the interaction of reflected shock wave with different distorted flame, which is supposed to be an interesting problem, and it's worth making a further study.

In the current study, we numerically studied the process of flame distortion of a spherical flame in shock accelerated flow using the reactive Navier-Stokes equations, and the main aim is to investigate the influence of different reflected shock wave distance on this process.

\section{Numerical Model}

Two-dimensional reactive N-S equations are employed to simulate the shock-flame interaction process. In our study, a splitting algorithm is applied, where the high accuracy five-point TVD scheme is utilized to approximate the numerical flux of inviscid term, and a second-order central difference scheme is used to discretize the viscous term. Besides, a second-order Runge-Kutta time marching is adopted for unsteady problems. In consideration of a detailed reaction mechanism needs tremendous computational resource, hence, one-step Arrhenius chemical reaction is employed for describing the combustion process. The detail of the numerical model can be referred in our previous works $[5,6]$. 


\section{Results and Discussions}

\section{Numerical validations}

The computational setup is shown in fig. 1 , where the spherical flame with density of $\rho_{1}=0.01578 \mathrm{~kg} / \mathrm{m}^{3}$ is surrounded by gas mixture $\mathrm{C}_{2} \mathrm{H}_{4} / 3 \mathrm{O}_{2} / 4 \mathrm{~N}_{2}$ with initial conditions of $\rho_{0}=0.1615 \mathrm{~kg} / \mathrm{m}^{3}, T_{0}=293 \mathrm{~K}$ at $p_{0}=13.3 \mathrm{kpa}$. The incident shock wave with mach number 1.7 initially locates at $x=0.012 \mathrm{~m}$, and then moves from left to right to interact with the spherical flame, and when it reflects from the right ending wall, the reflected shock wave will interact with the distorted flame again. The uniform grid size in this paper is $\Delta x=\Delta y=0.0475 \mathrm{~mm}$, and the resolution can fully correctly capture the evolution of the flame structure. In order to simplify the calculation, an axial symmetrical boundary condition is utilized at the bottom $(y=0 \mathrm{~m})$ of computational domain.

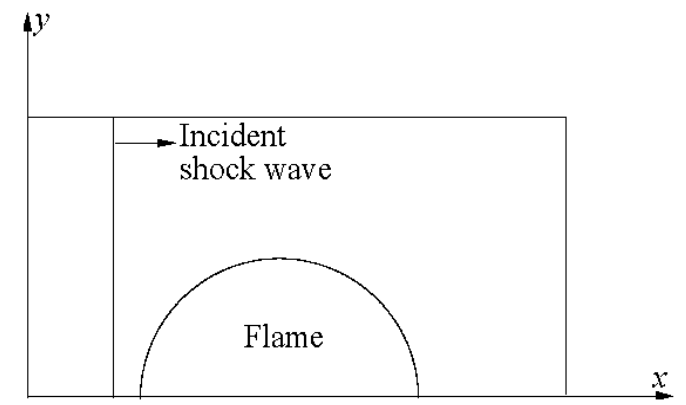

Fig. 1 Computational domain and initial flow field

The accuracy and reliability of numerical model have been proved valid by contrasting with the experiment described by Thomas et al. [4]. Specifically, fig. 2 gives the comparisons between experimental schlieren images and computational schlieren results at the selected times. Figures $2 \mathrm{a}$ and $2 \mathrm{~b}$ firstly show the scenarios of incident shock wave and spherical flame interaction, and it can be found that the spherical flame distorts via RM instability behind the incident shock wave, and forms a pair of longitudinal symmetrical flame. Moreover, figs. $2 \mathrm{c}$ and $2 \mathrm{~d}$ correspond to the interaction of reflected shock wave with flame. Actually, gas behind the reflected shock wave is stagnant with high temperature and pressure, which could promote the flame burning and expansion, hence, the distorted flame expands quickly, and meanwhile flips up and down to the wall. Not hard to see that good qualitative agreements of distorted flame and waves evolutions are obtained, which indicate the reliability of the numerical model in our study.
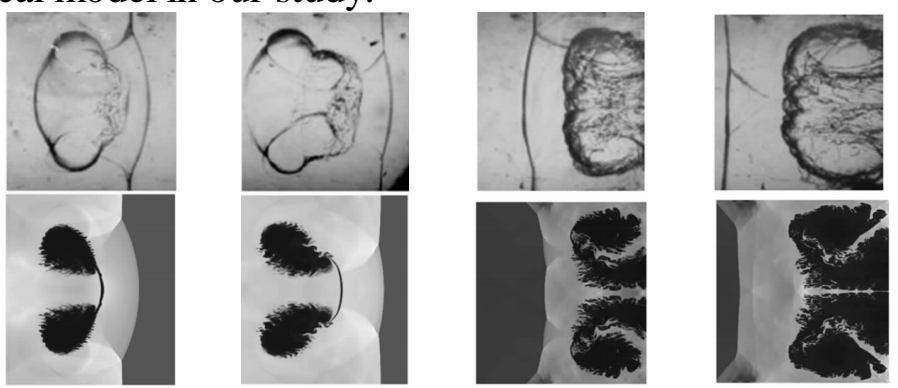

(a) $t=135.4 \mu \mathrm{s}$ (b) $t=188.8 \mu \mathrm{s} \quad(\mathrm{c}) t=447.3 \mu \mathrm{s} \quad(\mathrm{d}) t=500.7 \mu \mathrm{s}$

Fig. 2 Comparisons between experimental and computational results

\section{Visualized computational results}

Obviously, different shock reflected distance can induce the interaction of reflected shock wave with different distorted flame, and then more or less affects the flow field characteristics. To clarify the influence of different reflected distance on distorted flame evolution, table 1 shows five computational cases with different domain length. And the necessary meshes are also listed. It's easy to found that the grid size is the same with that in sec. 3.1. Besides, case 3 corresponds to the experiment setup of Thomas et al. [4]. 
Table 1 Computational parameters used in our paper

\begin{tabular}{ccc}
\hline Case & Domain $(x \times y) / \mathrm{mm}$ & Mesh \\
\hline 1 & $95 \times 38$ & $2000 \times 800$ \\
2 & $134.9 \times 38$ & $2840 \times 800$ \\
3 & $169.9 \times 38$ & $3576 \times 800$ \\
4 & $199.5 \times 38$ & $4200 \times 800$ \\
5 & $223.3 \times 38$ & $4700 \times 800$ \\
\hline
\end{tabular}

Figures 3 and 4 present the density contours of different cases at the selected times before and after the interaction of reflected shock wave with distorted flame. In each figure, computational results from top to down correspond to cases 1-5 respectively. It can be seen that the longer of the reflected shock wave distance, the more time is needed for shock waves travel, and distorted flames get more adequate development. It's obvious that flames in case 1and 2 are not fully developed. And with the increase of reflected shock wave distance, flame areas show an increase gradually in fig. 3. After a fixed time of $209.3 \mu \mathrm{s}$, reflected shock wave has passed through the distorted flame. Due to the high temperature and pressure behind reflected shock wave, differences of flame evolution become greater. For example, with the increase of reflected shock wave distance, the distorted flames stretch longer, and flames are more close to the top wall and bottom symmetrical axis.

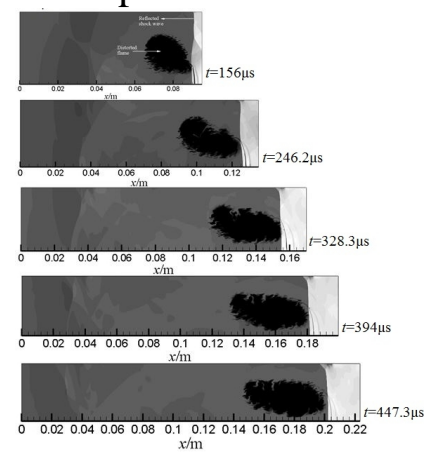

Fig. 3 Density contours before the interaction of reflected shock wave with flame

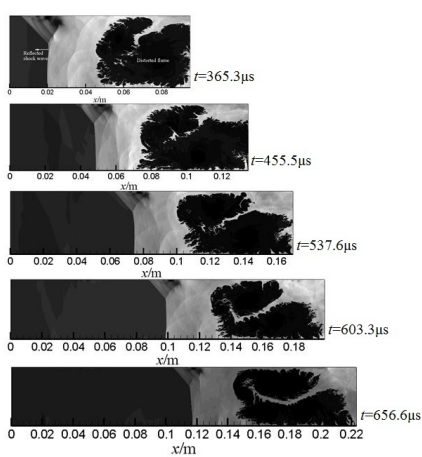

Fig. 4 Density contours after the interaction of reflected shock wave with flame

\section{Quantitative analysis}

In order to study the evolution of distorted flame in different cases, integrated variables are adopted for quantitative analysis. The valid flame area $A$ and mean heat release rate $\mathrm{d} h / \mathrm{d} t$ are defined as follows:

$$
\begin{aligned}
& A=\int_{D}(1-X) \mathrm{d} A \\
& \frac{d h}{d t}=\frac{\int_{F} \rho \cos x \mathrm{~d} A}{A}
\end{aligned}
$$

where the subscripts $D$ and $F$ represent the entire computational domain and flame zone (reactant mass fraction $Y \leq 0.99$ ) respectively, $X$ is the local volume fraction of fuel, $\rho$ is the density, of is the chemical reaction rate, and $q$ is the heat production of reaction per unit mass.

Fig. 5 presents the time histories of dimensionless valid flame area $A / A_{0}$ ( $A_{0}$ is the initial flame area) and mean heat release rate. It is found that the change of flame area experiences four stages in sequence, that is, incident shock wave compressed stage (I), flame expanding stage (II) for the first time behind incident shock wave, reflected shock wave compressed stage (III), and flame expanding stage (IV) for the second time. Especially for stage IV, owing to the gas behind the reflected shock wave is stagnant with high temperature and pressure, the flame can greatly expand. From the view of mean heat release rate, it continues to grow in compressed stages I and III, and maintains a high fixed level in IV stage. It is not hard to found that the different reflected shock wave distance changes the interaction times of reflected shock wave with flame, hence, the rules of flame area and mean heat release rate evolution of different cases appear a kind of change in stages III and IV. It must be noted that mean heat release rate 
in stage IV enhances one magnitude than that in stage II, which implies the huge boost of reflected shock wave on combustion.

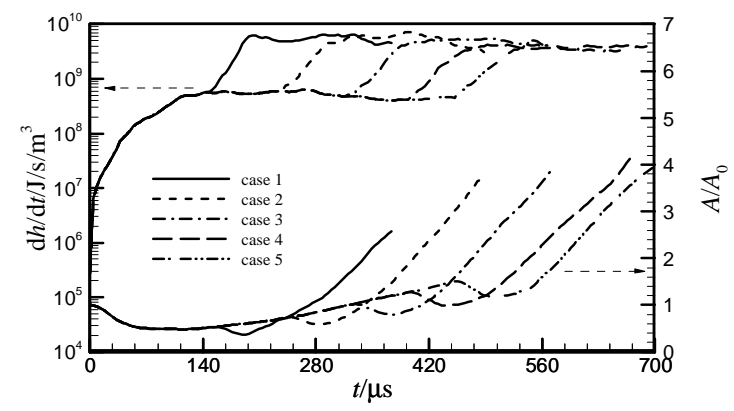

Fig. 5 Time histories of valid flame area and mean heat release rate for different cases

When shock wave interacts with flame, it can induce the instability of flame interface owing to the RM instability. Also, vortices are generated at the interface, which are mainly affected by baroclinic effect, and vortices could promote the mixing of flame and fresh fuel. We had already discussed the vortices evolution of distorted flame in our work [5]. In this study, different reflected shock wave distances are considered to determine if there are any variations. Fig. 6 shows the time histories of average vortices, which are computed using the following formula:

$$
\omega_{\text {ave }}=\frac{\int_{F} \sqrt{\omega^{2}} \mathrm{~d} A}{\int_{F} \mathrm{~d} A}
$$

where $\omega^{2}$ represents the enstrophy, $\omega^{2}=\omega_{x}^{2}+\omega_{y}^{2}$, here $\omega_{x}$ and $\omega_{y}$ are the vorticity components in $x$ and $y$ directions of flow field, respectively. It can be seen that all the cases have a same variation tendency, which is in accord with our previous work [5]. However, due to the viscosity of the flow field, the peak value (mainly appears in stage III) is getting smaller and smaller with the increase of reflected shock wave distance. In this situation, longer distance means longer time for the evolution of distorted flame. Thus, the influence of viscosity becomes more significant.

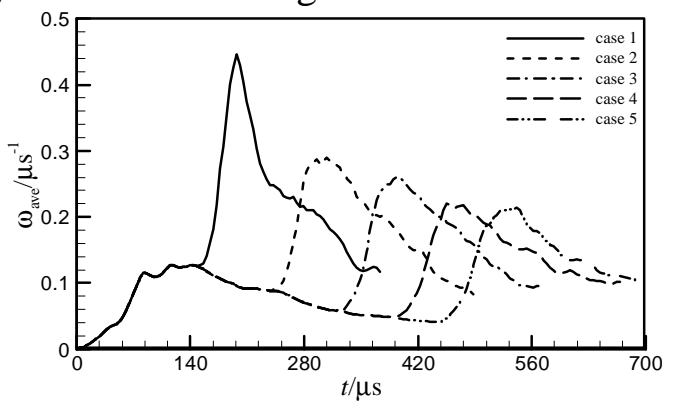

Fig. 6 Time histories of average vortices for different cases

As well known, the instability and vortices of wrinkled flame interface could promote the flame and fuel mixing and further increase the total heat release. To study the influence of interfacial morphology and geometrical self-similarity on combustion process, fig. 7 gives the time histories of interface length $L / L_{0}\left(L_{0}\right.$ is initial flame interface length) and fractal dimension $D_{m}$ of flame interface, where $D_{m}$ is obtained by 'boxing counting' method [7]. And $D_{m}$ value represents the wrinkled degree of flame interface. It is found that before the interaction of reflected shock wave with flame, the interface length and fractal dimension both increase, which imply that the small-scale wrinkles of flame interface are dominant, but in the later stage II, the fractal dimension maintains 1.3 around while the interface length continues growing, which means large-scale flame deformation accelerates the interface length. Hence, physical process is predominant in stages I and II. While after the reflected shock wave interacts with distorted flame, the flame zone is in a state with high temperature and pressure, reaction rate increases, and combustion process accelerates. So, in general, there mainly exist two factors which could influence the interface length and fractal dimension, that is, one is reflected shock wave compression ( $D_{m}$ value increases), and the other one is rapid combustion process $\left(D_{m}\right.$ value decreases). In stages III and IV, all $D_{m}$ values increase firstly due to the reflected shock wave compression, and then decrease owing to the rapid combustion process. Moreover, in the case of long reflected shock wave distance, 
distorted flame is more close to the wall (according to fig. 4), thus $D_{m}$ value decreases. However, the comprehensive result is that interface length almost increases in every case, even in the period of fractal dimension decreases, which implies that chemical reaction plays an important role at this time (combustion promotes the flame expands, hence, the interface length increases, while $D_{m}$ value decreases means the wrinkles reduces, hence, the interface length decreases). It's worth noting that the peak of fractal dimension of case 5 is the smallest owing to the longer time of distorted flame in the flow filed, so the influence of viscosity is enhanced.

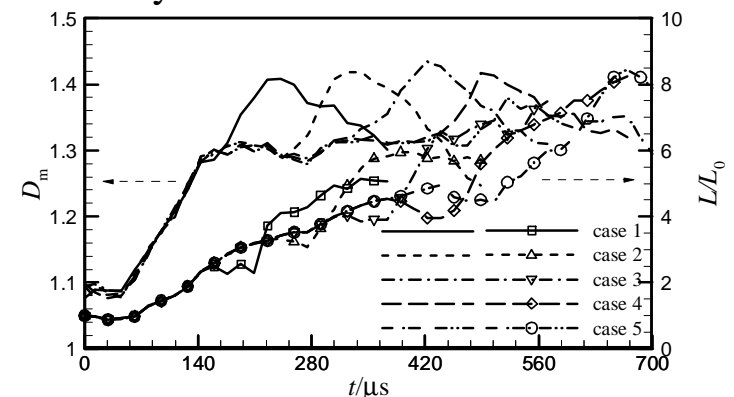

Fig. 7 Time histories of interface length $L / L_{0}$ and fractal dimension $D_{m}$ of flame interface

\section{Conclusions}

In present study, the two dimensional flame distortion and instability induced by incident shock and its reflected waves is numerically studied for an ethylene/oxygen/ nitrogen mixture. In particular, five different reflected shock wave distances are considered. The results show that the flame is compressed firstly, and then expands after every shock impact. Before the reflected shock wave compression, physical process is dominant. While after the reflected shock wave compression, mean heat release rate enhances one magnitude, and the enhancement of chemical reaction could forbid the flame interface wrinkles thereby playing an important role at this time. Moreover, different reflected shock wave distances induce different flame morphologies, and the longer of the distance, the influence of viscosity is stronger, which also forbids the flame interface wrinkles.

\section{Acknowledgements}

This work was financially supported by the National Natural Science Foundation of China (Grant No. 11402102), the Natural Science Foundation of Jiangsu Province of China (Grant No. BK20140524), and the National Science Foundation for Post-doctoral of Jiangsu Province of China (Grant No. 1402013B).

\section{References}

[1] J. Yang, T. Kubota, and E.E. Zukoski: AIAA Journal Vol. 31(5) (1993) p. 854.

[2] E.S. Oran, and V.N. Gamezo: Combustion and Flame Vol. 148 (2007) p.4.

[3] Y. Ju, A. Shimano, and O. Inoue: 27th Symposium (International) on Combustion, The Combustion Institute, Pittsburgh, USA (1998) p.735

[4] G.O. Thomas, R. Bambrey, and C. Brown: Combustion Theory and Modelling Vol. 5(4) (2001) p. 573

[5] Y.J. Zhu, G. Dong, and Y.X. Liu: Combustion Science and Technology Vol. 185(10) (2013) p. 1415

[6] Y.J. Zhu, G. Dong, Y.X. Liu, B.C. Fan, and H. Jiang: Acta Mechanica Sinica Vol. 29(1) (2013) p. 54

[7] H.D. Ng, H.A. Abderrahmane, K.R. Bates, and N. Nikiforakis: Communications in Nonlinear Science and Numerical Simulation Vol. 16(11) (2011) p. 4158 